\title{
Economic Operation of Interconnected Power System and Unidirectional Flow through GSA
}

\author{
Anumeha \\ Department of Electrical \\ Engineering \\ National Institute of \\ Technology, Jamshedpur, \\ India
}

\author{
K. B. Yadav \\ Department of Electrical \\ Engineering \\ National Institute of \\ Technology, Jamshedpur, \\ India
}

\author{
S. Agrawal \\ Department of Electrical \\ Engineering \\ National Institute of \\ Technology, Jamshedpur, \\ India
}

\begin{abstract}
This paper introduces a GSA for implemented to economic operation of a interconnected area power system and computes how much power has to be generated internally in an area and how much power has to be borrowed from other area through tie-line for a specified load so that generation cost is minimized in most economical sense. This method is explained with an example and the result obtained by the proposed method is compared with by particle swarm optimization (PSO) as reported in literature. It has been shown that this method is more efficient and takes less computation time than PSO.
\end{abstract}

\section{Keywords}

Optimization, PSO, GSA, ELD, Power system

\section{INTRODUCTION}

In early stage each unit were serving a restricted local area. It has got its own restrictions such as reliability, quality of service, security, spinning reserve capacity. Keeping in view of these tribulations the concept of interconnection of different generating units forming an area (region) developed where all the generating units are working in unison. In an area there are a number of generating units, each has different cost characteristic. The problem arises that for a given load what should be the generation of each unit (operating under given constraints) so that the total cost involved is minimum known as Economic Load Dispatch (ELD) problem. This is computed and controlled by a power system grid and the information is sent to different generating units. The solution of this problem is found in standard books [1-2] and reputed journals [3-5]. Various mathematical programming methods has been applied to solve this problem such as LP (Linear Programming) [6-7] where all the constraints \& objective function are linear, Non Linear Programming (NLP) [8], Dynamic Programming (DP) [9-10]. The DP is suffering from the problem of "curse of dimensionality". Computational intelligence techniques based heuristic methods are also developed for solution of such problems e.g. Particle Swarm Optimization (PSO) [11-15], Genetic Algorithm (GA) [16], Artificial Neural Networks (ANN) [17] etc.

The load curve of different area of power system is of different shape. The peak load of different area occurs at different time in a day. The load can be transferred from one area to other, depending on the power generating capacity and load demand of each area and their difference, through tie line between the two areas resulting in a co-ordinate operation of the power system. It improves the load factor, reduces the spinning reserve of each area, and improves the power system security, reliability and power quality. Recently a paper has been published [18] considering power transfer from one area having a number of generating units to other on the basis of multiple flat rate cost depending on the quantity of power transfer. The solution method proposed was a conventional method of Lagrange multiplier. Recently intelligence based optimization method (PSO) has also been applied to solve this problem [19].

Recently a new Meta heuristic method for optimization known as GSA is developed by E. Rashedi et al. in 2009 based on the Newton's laws of gravity and motion [20]. This method has been applied to a number of fields. A few has been pointed out in the field of power system optimization and control such as ELD problem [21-24], Automatic Generation Control (AGC) [25]. A review by Sabri et al. of the GSA method is presented in [26].

In this paper, the GSA (intelligence based heuristic optimization method) is implemented for economic operation of a two area power system. The advantage of the method is that it is applicable to both linear, nonlinear or piece wise linear systems as in case of PSO. The method is explained by taking an example having two generating machines in an area interconnected to another area by tie line. The results obtained by GSA are compared with the conventional method [18] and PSO [19]. It has been shown that GSA outperforms the PSO evolutionary optimization method with respect to computation time.

\section{GRAVITATIONAL SEARCH ALGORITHM (GSA)}

The Newton's law of gravitation states that each particle attracts every other particle with a force known as gravitational force of attraction. This force is proportional to the product of the mass of the particles and inversely proportional to distance between them. E. Rashedi et al. has developed a new Meta heuristic optimization method in 2009 on the basis of this natural phenomenon, known as Gravitational Search Algorithm. The optimization method is presented in brief as follows.

A set of agents ' $\mathrm{n}$ ' (as no. of particles in PSO) each having a mass $M_{i}$ considered. The position of each mass $M_{i}$ is represented by

$\mathrm{X}_{\mathrm{i}}=\left(\mathrm{x}_{\mathrm{i} 1}, \mathrm{x}_{\mathrm{i} 2},--------, \mathrm{x}_{\mathrm{im}}\right.$ for $\mathrm{i}=1,2,---, \mathrm{n}$

Where $\mathrm{n}$ is the number of agents and $\mathrm{m}$ is the dimension (number of variables) of each agent or mass (search space). The mass of each agent at current $\left(k^{\text {th }}\right)$ iteration depends upon the fitness function as follows: 


$$
\begin{aligned}
& \mathrm{Q}_{\mathrm{I}}^{\mathrm{k}}=\frac{\text { fit }_{i}^{k} \text {-worst }}{{ }_{i}^{k}} \\
& \text { best }^{k}-\text { worst }^{k} \\
& \mathrm{M}_{\mathrm{i}}^{\mathrm{k}}=\frac{Q_{i}^{k}}{\sum_{\mathrm{i}=1}^{\mathrm{k}} \mathrm{Q}_{\mathrm{i}}}
\end{aligned}
$$

Where $k$ is the iteration count, $M_{i}^{k}$ and $f i t_{i}^{k}$ represent the mass and fitness value of $i_{t h}$ agent at $k^{\text {th }}$ iteration respectively. For minimization problem best $t^{k}$ and worst $^{k}$ are defined as

$$
\begin{aligned}
& \text { best }^{k}=\min _{i \in 1 \text { to } n}\left\{f i t_{i}^{k}\right\} \\
& \text { worst }^{k}=\max _{i \in 1 \text { to } n}\left\{\text { fit }_{i}^{k}\right\}
\end{aligned}
$$

The acceleration of an agent is computed by considering total forces from a set of heavier masses that apply on it based on the Newton's law of gravity. The total forces can be computed as given in equation (6)

$F_{i j}^{k}=\sum_{l \in k b e s t, l \neq i} \operatorname{rand}_{i} G^{k} \frac{M_{l}^{k} M_{i}^{k}}{R_{i l}+\delta}\left(x_{l j}^{k}-x_{i j}^{k}\right)$

Where rand is uniformly distributed random number in the interval $(0$ and 1$) . \delta$ is a constant small value. Ril is the Euclidean distance between two agents ' $\mathrm{i}$ ' and ' 1 ' represented in equation (7). The gravitational constant at iteration $\mathrm{k}$ is represented by equation (8).

$$
\begin{aligned}
& \text { Rilk }=\|X i k, X l k\| 2 \\
& G^{k}=G_{0} e^{-\beta \frac{k}{k_{m}}}
\end{aligned}
$$

Where $G_{0}$ is initial value of gravitational constant and $K_{m}$ is the maximum number of iterations specified and $\beta$ is constant.

Here kbest is the set of first $k$ agents with the best fitness value and biggest mass which is a function of iteration $k$ (time) initialized to $k_{0}$ at the beginning and decreasing with iteration $\mathrm{k}$ (time). Here $k_{0}$ is set to $n$ (total number of agents) and is decreasing linearly to 1 .The acceleration of an agent $i$ in $j^{\text {th }}$ dimension $\left(\alpha_{i j}{ }^{k}\right)$ can be computed using equation (6) and given as:

$$
\alpha_{i j}^{k}=\frac{F_{i j}^{k}}{M_{i}^{k}}=\sum_{l \in k \text { best }, l \neq i} \operatorname{rand}_{i} G^{k} \frac{M_{l}^{k}}{R_{i l}+\delta}\left(x_{l j}^{k}-x_{i j}^{k}\right)
$$

Once the acceleration of an agent is computed, the velocity of the agent is updated by equation (10).

$V_{i j}{ }^{k}+1=\operatorname{rand} * V_{i j}{ }^{k}+\alpha_{i j}{ }^{k}$

Now the position of the agent can be updated by adding the velocity to the $k^{\text {th }}$ position equation (11).

$X_{i j}{ }^{k}+1=x_{i j}{ }^{k}+V_{i j}{ }^{k}+1$

In the next section the ELD problem and the problem faced while solving this problem by GSA method is discussed.

\section{PROBLEM STATEMENT}

A two area (grid) power system interconnected through tie line represented in Figure 1. In area 1(grid1) there are ' $N$ ' generating units interconnected through loss less power lines is assumed and it is interconnected through tie-line to other area(grid). It is also assumed that there is always power available in area 2(grid2) to be transferred as and when required by area 1 and power transmission loss is negligible.

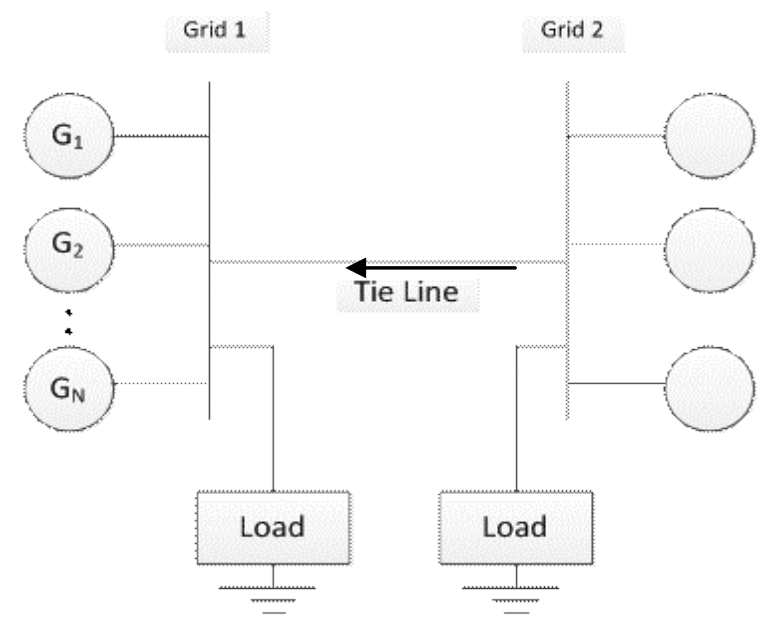

Figure 1: System representation.

The power borrowed from area 2 (grid) is represented by Pt. It is assumed that the power generating cost (operating cost) of each unit depends upon its own generation and is represented as

$C P_{i}=a_{i}+b_{i} \cdot P_{i}+c_{i} \cdot P_{i}^{2}$

Where $a_{i}, b_{i}$ and $c_{i}$ are constants depending on each unit, $P_{i}$ is power generation of $i^{\text {th }}$ unit and $C P_{i}$ is the operating cost of $i^{\text {th }}$ unit. However the operating cost represented in equation (12) may contain additional smooth/non-smooth nonlinear terms e.g. valve-point effect and change of fuel. It is assumed that power can also be borrowed from other area(grid) through tie line may be during peak load hours or otherwise when it is required for economic operation of composite power system, It is further assumed that the cost of borrowed power from other area(grid) is on the flat rate basis. Let the maximum power that can be borrowed is $P_{\text {tmax }}$ and the cost of power transfer is on two slabs, where the cost per unit of power transfer from 0 to $P_{t l}$ is $\lambda_{1}$ and $\mathrm{P}_{\mathrm{t} 1}$ to $P_{t \operatorname{tmax}}$ is $\lambda_{2}$. The tie line power cost can be represented as in (13).

$$
\begin{array}{lr}
C_{t i e}=\lambda_{l} \cdot P_{t} & \text { for } P_{t}<P_{t l} \\
C_{t i e}=\lambda_{l} \cdot P_{t 1}+\lambda_{2} \cdot\left(P_{t}-P_{t l}\right) & \text { for } P_{t 1}<P_{t}<P_{t m a x}
\end{array}
$$

The total cost of internal generation and tie line power is given by

$$
C_{\text {total }}=\sum_{i=1}^{N} C P_{i}+C_{\text {tie }}
$$

Now the problem can be stated as:

For a given load $P d$ find the power generation of each unit (Pi) and tie line power $\left(P_{t}\right)$ borrowed from other area(Grid) ,such that the total cost given by (14) is minimum subject to the following constraints:

i)

Equality constraints

$$
\sum_{i=1}^{N} P_{i}+P_{t}=P d
$$

$$
\text { Inequality Constraints }
$$

The power generation of each unit is limited by maximum power $P_{\max }$ and minimum power $P_{\min }$ as represented in (16)

Pmin $i<P_{i}<P \max i$

$$
0<P_{t}<P_{\text {tmax }}
$$


This problem has been solved by using LaGrange method [18] and PSO a Meta heuristic method [19]. A more efficient Meta heuristic method GSA has been developed recently as discussed in section 2. In this paper it is proposed to solve this problem using GSA method as discussed in next section.

\section{SOLUTION PROCEDURE}

The interconnected multiple area (grid) power system optimization problem is stated in the previous section. In this section this problem is solved by GSA. It is a heuristic iterative method revolving around two equations velocity (distance) and position updating given by the two equations (10) and (11). The solution procedure is presented below.

\section{A. Initialization}

Position: To start the iteration process initialization of individual agent's position and velocity is necessary. At first a population size (number of agents) $\mathrm{n}$ is selected. Next the number of variables $m$ is identified as per the problem. Then each particle's position is selected randomly wide spread in the search space such that it does not violate the constraints represented by equations (15) \& (16) for $i^{\text {th }}$ agent as $x i=$ (xi10, xi20,.......,xim0 ) in $\mathrm{m}$ dimensional space where $\mathrm{m}$ is the number of variables. In this case $\mathrm{m}$ is the power generating units and the last variable is the tie line power.

Velocity: The initial velocity of agent $\mathrm{i}$ is assumed as $\mathrm{Vi}=$ (Vi10, Vi20,......,Vim0).

\section{B. Updating Velocity and Position}

In order to modify the position of each agent in iteration, it is necessary to compute the modified velocity in that iteration by equation (10). The modified velocity is computed by computing the acceleration by equation (9).

After updating the position by equation (11), it is necessary to check the inequality constraints as follows:

$$
\begin{array}{lc}
\mathrm{x}_{\mathrm{ij}}{ }^{\mathrm{k}}+\mathrm{V}_{\mathrm{ij}}{ }^{\mathrm{k}+1} & \text { if } \mathrm{x}_{\mathrm{ij} \min }<\left(\mathrm{x}_{\mathrm{ij}}{ }^{\mathrm{k}}+\mathrm{V}_{\mathrm{ij}}{ }^{\mathrm{k}+1}\right)<\mathrm{x}_{\mathrm{ij} \max } \\
\mathrm{x}_{\mathrm{ij}}{ }^{\mathrm{k}+1}=\mathrm{x}_{\mathrm{ij} \text { mi }} & \quad \text { if }\left(\mathrm{x}_{\mathrm{ij}}{ }^{\mathrm{k}}+\mathrm{V}_{\mathrm{ij}}{ }^{\mathrm{k}+1}\right)<\mathrm{x}_{\mathrm{ij} \min } \\
\mathrm{x}_{\mathrm{ij} \max } & \text { if }\left(\mathrm{x}_{\mathrm{ij}}{ }^{\mathrm{k}}+\mathrm{V}_{\mathrm{ij}}{ }^{\mathrm{k}+1}\right)>\mathrm{x}_{\mathrm{ij} \max }
\end{array}
$$

In addition to the inequality constraints as in (16) the equality constraint given by (15) has to be satisfied for implementing the GSA algorithm. To satisfy the equality constraints a heuristic method is proposed as given below.

i) Find the sum of the variables of a particle (sum of generations of each machine in this case)

ii) Compare it with the equality constraint and find the difference.

iii) The difference is divided by the number of variables and then adds this value to each element of the particle.

\section{a. Stopping Citerion}

The proposed iterative method is terminated if the iteration approaches a predefined criterion, usually a sufficient good fitness in this problem absolute value of (best-worst) or if it reaches the maximum number of iterations as defined.

The problem stated in previous section can be solved using GSA method as explained in this section. Normally this method gives solutions to all types of problems it may be linear, continuous, discontinuous or nonlinear problems. In the next section the method is explained by taking an example. Complete solution steps are given in appendix- 1 .

\section{b. Example}

The example considered here for explaining the procedure is taken from reference, [18] as given below:

A two area (grid) power system connected through tie line is considered as shown in figure 1. Area 1(grid 1) has two generating units having cost characteristics as represented by equation (12).

$$
\mathrm{CP} 1=120+40 \mathrm{P} 1+0.1 \mathrm{P} 12
$$$$
\mathrm{CP} 2=100+32 \mathrm{P} 2+0.125 \mathrm{P} 22
$$

The tie line power cost is assumed to be in two slabs having per unit cost of Rs. $60(\lambda 1)$ for first $50 \mathrm{MW}$ (Pt1) and Rs. 65 ( $\lambda 2)$ per MW up to next $50 \mathrm{MW}$. So the maximum tie line power that can be borrowed is assumed to be $100 \mathrm{MW}$. The power generation limit of each generating unit is assumed as given below.

Unit 1: $5 \mathrm{MW}\left(\mathrm{P}_{\min 1}\right) \leq \mathrm{P}_{1} \leq 150 \mathrm{MW}\left(\mathrm{P}_{\max 1}\right)$

Unit 2: $5 \mathrm{MW}\left(\mathrm{P}_{\min 2}\right) \leq \mathrm{P}_{2} \leq 150 \mathrm{MW}\left(\mathrm{P}_{\max 2}\right)$

Tie-line power: $0 \leq \mathrm{P}_{\mathrm{t}} \leq 100 \mathrm{MW}$

The objective function/ fitness function is given by the total cost as per equation (14)

$C_{\text {total }}=\mathrm{CP}_{1}+\mathrm{CP}_{2}+\mathrm{C}_{\text {tie }}$

Where,

$$
\begin{array}{lr}
C_{\text {tie }}=\lambda_{1} \cdot P_{t} & \text { for } P_{t} \leq 50 \mathrm{MW} \\
=\lambda_{1} P_{t 1}+\lambda_{2} \cdot\left(P_{t}-P_{t 1}\right) & \text { for } 50<P_{t} \leq 100
\end{array}
$$

The problem could be stated as for a given load $\mathrm{Pd}=232 \mathrm{MW}$ find the generation of each unit and tie line flow such that the cost function given by equation (14) is minimum. However, the inequality and equality constraints given by equation (15) and equation (16) should be satisfied. The constants assumed are $\delta=0.9, \beta=10$ and $\mathrm{G} 0=10$.

Maximum number of iteration $\mathrm{km}$ assumed for this problem is 1000. In this case there are three variables generation of unit 1 , generation of unit - 2 and tie-line flow. The number of objects or agents assumed is 4 . Now the initial position of the agents is selected satisfying the equality and inequality constraints equations (15) \& (16) respectively as indicated below.

The initial value of position and velocity are assumed as mentioned in Table I and Table II.

Table I: Initial value of position

\begin{tabular}{|c|r|r|r|}
\hline Agent No. & $\mathrm{P}_{1}$ & $\mathrm{P}_{2}$ & $\mathrm{P}_{\mathrm{t}}$ \\
\hline 1 & 20 & 124 & 88 \\
\hline 2 & 130 & 40 & 62 \\
\hline 3 & 70 & 120 & 42 \\
\hline 4 & 112 & 100 & 20 \\
\hline
\end{tabular}

Table II: Initial value of velocity

\begin{tabular}{|c|c|c|c|}
\hline Agent No. & $\mathrm{V}_{1}$ & $\mathrm{~V}_{2}$ & $\mathrm{~V}_{\mathrm{t}}$ \\
\hline 1 & 3 & 5 & 7 \\
\hline 2 & -2 & 8 & 10 \\
\hline 3 & 7 & -2 & 5 \\
\hline 4 & 10 & 0 & 5 \\
\hline
\end{tabular}


The stopping criteria assumed as $\mid\left(\right.$ best $t^{k}-$ worst $\left.^{k}\right) \mid<0.00001$.

\section{RESULTS}

The problem is solved for a load of $232 \mathrm{MW}$. The power generation of unit $1 \mathrm{P} 1$, unit $2 \mathrm{P} 2$ and tie-line power Pt is 100 MW, 112 MW and 20 MW respectively. The optimal cost is Rs. 11572 and computation time is 0.79347 seccond. Graph between worst Vs iterations and best Vs iterations is plotted Figure 2 to show the convergence. When both the plots coincide (best $=$ worst) the optimum value is reached. From the graph one can visualize that convergence towards end (near optimum) is very slow. The results computed by the present method compared with the results of PSO [19] for the same problem. It is observed that the generation of unit 1 , unit 2 , tie- line power and the optimum cost is same in both the methods. However, the computation time of GSA method is lower of the order of 0.08 seconds compared to PSO method which is approximately 6 seconds. It shows that the GSA method is more efficient then PSO. Since computation time is low in GSA, it can be implemented on-line.

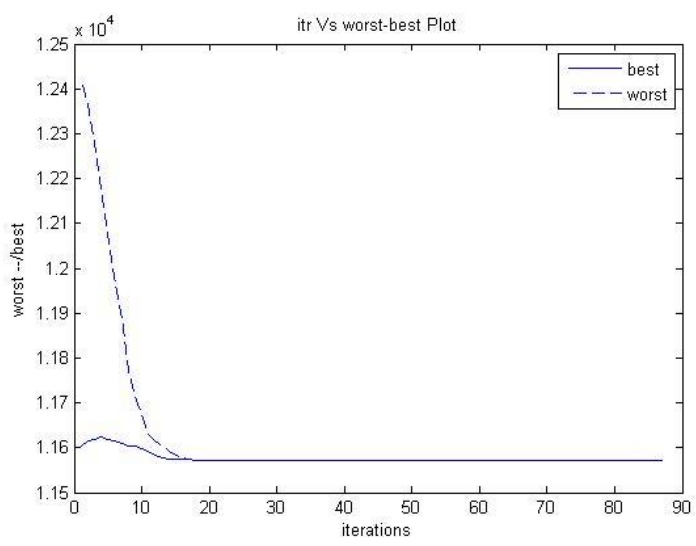

Figure 2: Graph Worst/Best Vs iterations

\section{CONCLUSION}

The economic operation of two area (Grid) power system by GSA is presented. The results obtained by this method compared with PSO. It has been shown that the computation time by the present method quite lower than the computation time by PSO for the same problem [19]. So the GSA method of solution is more efficient than PSO. As the computation time is low in GSA, it can be implemented on-line It has been pointed out that the convergence of GSA is quite slow towards end (near the optimum value) Figure 2. The computation time could be further reduced by improving the convergence by using acceleration factor or by hybridization with some other method.

\section{REFERENCES}

[1] Olle I. Elgared, "Electric Power System Theory, An Introduction", Second Edition, TMH, New Delhi, 1983.

[2] D. P. Kothari and I. J. Nagrath, "Modern Power System Analysis", Third Edition, TMH, New Delhi, 2003.

[3] A. K. Ayoub and A. D. Patton, "Optimal Thermal generating Unit Commitment", IEEE Trans on Power Apparatus and Systems, vol. 90, no. 4, pp 1752-56, July 1971.

[4] H. H. Happ, "Optimal Power Dispatch - a Comprehensive Survey", IEEE Trans. on Power Apparatus and Systems, vol. 96, no. 3, pp. 841-854, May 1977.
[5] IEEE Committee Report, "Present Practices in the Economic Operation of Power System", IEEE Trans on Power Apparatus and Systems , vol. 90, 1971.

[6] A. Farag, S. Al-Baiyat, and T. Cheng, "Economic Load Dispatch Multi Objective Optimization Procedures Using Linear Programming Technique”, IEEE Trans. on Power System, vol. 10, no. 2, pp 731 - 738, May 1995.

[7] R. Jabr, A. Coonick and B. Cory, "A Homogeneous Linear Programming AlgorithmFor the Security Constrained Economic Dispatch Problem", IEEE Trans. on Power System, vol. 15, no. 3, pp. 930-936, Aug 2000.

[8] S. Virmani, E. Adrian et. al. "Implementation of a Lagrangian Relaxation based Unit Commitment Problem”, IEEE Trans. on Power System,, vol. 4, no. 4, pp1373-1380, Nov 1989.

[9] Z. Oyang and S. Shahidehpour, “An Intelligent Dynamic Programming for Unit Commitment Application”, IEEE Trans .on Power System, vol-6, no. 3, pp 1203-1209, Aug 1991.

[10] W. Hobbs, G. Hermon et. al. "An Enhanced Dynamic Programming Approach for Unit Commitment “, IEEE Trans. on Power System, vol. 3, no. 3, pp. 1201-1205, Aug. 1988.

[11] M. Sudhakaran et. al. "Particle Swarm Optimization for Economic and Emission Dispatch Problem", Institute of Engineers, India, vol. 88, pp. 39-45, June 2007.

[12] Y. Shi and R. C. Eberhart, "Particle Swarm Optimization: Developments, Applications and Resources", Proceedings of the 2001 Congress on Evolutionary Computation, vol. 1, pp. 81-86, May 2001.

[13] R. C. Eberhart and Y. Shi, "Comparing Inertia Weights and Constriction Factor in Particle Swarm Optimization", Proceedings of the 2000 Congress on Evolutionary Computation, vol. 1, pp. 84-88, June 2000.

[14] J. Kennedy and R. Eberhart,"Particle Swarm Optimization" Proc. Int. Conf. Neu-Ral Networks (ICNN), vol. 4, pp. 1942-1948, Nov 1995.

[15] Yamille del Valle et.al. "Particle Swarm Optimization : Basic Concepts, Variants And Applications in Power Systems", IEEE Trans. on Evolutionary Computation, vol. 12, no. 2, pp. 171-195, Apr 2008.

[16] Barkirtzis, V. Petridis and S. Kazarlis, "Genetic algorithm Solution to the Econo-mic Dispatch Problem", IEE Proceedings-Generation, Transmission, and Distribution, vol. 141, no. 4, pp. 377-382, July 1994.

[17] S. Senthil Kumar and V. Palanisamy, "A Fast computation Hopfield Neural Net-Work Method of Unit Commitment”, Institute of Engineers India, vol. 88, pp. 3-9, June 2007

[18] S. Agrawal, S. K. Das and D. Mazumdar, "A Novel Approach to Unit commit-Ment and Tie Line Power Flow for multiple area Power System", MIT International Journal of Electrical and Instrumentation Engineering, vol. 1, no. 2, pp. 70-75, Aug 2011.

[19] S. Agrawal, T. Bakshi and D. Mazumdar, "Optimal Power Pooling for a Multiple Area Power System through PSO”, International Journal on Electrical Engineering and Informatics, vol. 6, no. 2, pp. 280-296, June 2014. 
[20] E. Rashedi et. al.,"GSA: a Gravitational Search Algorithm", Information Sciences, vol.179, no. 13, pp. 2232-2248, June 2009.

[21] R. K. Swain et. al., "Gravitational Search Algorithm for Optimal Economic Dispatch", Procedia Technology, vol. 6, pp. 411-419, 2012.

[22] P. K. Roy, "Solution of Unit Commitment Problem using Gravitational Search Algorithm", International Journal of Electrical Power \& Energy Systems, vol. 53, pp. 85-94, Dec 2013.

[23] B. Shaw et al., "A Novel Optimization based Gravitational Search Algorithm for Combined Economic and Emission Dispatch Problem of Power System",
International Journal of Electrical Power \& Energy Systems, vol. 35, no. 1, pp. 21-33, Feb 2012.

[24] S. Duman et. al., "Gravitational Search Algorithm for Economic Dispatch with Valve- Point Effect", International review of Electrical Engineering, vol. 5, no. 6, pp. 2890-2895, Dec 2010.

[25] U. K. Rout et. al., "Gravitational Search Algorithm based Automatic Generation control for Interconnected Power System", International Conerence on Circuits, Power and Computing Technologies (ICCPCT-2013), pp.558-563, Mar 2013.

[26] N. M. Sabri et. al. "A Review of Gravitational Search Algorithm”, Int. J. of Advance. Soft Comput. Appl., vol. 5, no. 3, pp. 1-39, Nov. 2013. 